\title{
Second Hankel Determinant for a Subclass of Alpha Convex Functions
}

\section{Gagandeep Singh ${ }^{1 *}$ and Gurcharanjit Singh ${ }^{2}$}

${ }^{1}$ Department of Mathematics, M.S.K. Girls College, Bharowal (Tarn-Taran), Punjab, India

${ }^{2}$ Department of Mathematics, Guru Nanak Dev University College, Chungh (Tarn-Taran), Punjab, India

\begin{abstract}
In this paper a sharp upper bound of second Hankel determinant $\left|a_{2} a_{4}-a_{3}^{2}\right|$ for the functions belonging to the class $M_{\alpha}(A, B)$ of alpha convex functions is established. The class of alpha convex functions is an extended version of the classes of starlike functions and convex functions. By giving the particular values to alpha, it is easy to obtain the results of starlike and convex functions. The class discussed in this paper is an extended version of the class of alpha convex functions. By giving the particular values to $A$ and $B$, the result of alpha convex functions can be easily obtained.
\end{abstract}

Keywords: Analytic functions; Starlike functions; Convex functions; Alpha convex functions; Subordination; Schwarz function; Second Hankel determinant

\section{Introduction}

Let $A$ be the class of analytic functions of the form

$$
f(z)=z+\sum_{k=2}^{\infty} a_{k} z^{k}
$$

in the unit $\operatorname{disc} E=\{z:|z|<1\}$.

By $S$ we denote the class of functions $f(z) \in A$ and univalent in $E$.

Let $U$ be the class of Schwarzian functions

$$
w(z)=\sum_{k=1}^{\infty} d_{k} z^{k}
$$

which are analytic in the unit $\operatorname{disc} E=\{z:|z|<1\}$ and satisfying the conditions $w(0)=0$ and $|w(z)|<1$.

Let $f$ and $g$ be two analytic functions in $E$. Then $f$ is said to be subordinate to $g$ (symbolically $f \prec g$ ) if there exists a Schwarz function $w(z) \in U$, such that $f(z)=g(w(z))$.

$M_{\alpha}(A, B)$ denote the subclass of functions $f(z) \in A$ and satisfying the condition

$$
\frac{z f^{\prime}(z)+\alpha z^{2} f^{\prime \prime}(z)}{(1-\alpha) f(z)+\alpha z f^{\prime}(z)} \prec \frac{1+A z}{1+B z},-1 \leq B<A \leq 1,0 \leq \alpha \leq 1, z \in E .
$$

The class $M_{\alpha}(A, B)$ is the subclass of alpha-convex functions. The following observations are obvious:

$M_{\alpha}(1,-1) \equiv M(\alpha)$, the class studied by Singh [1].

$M_{0}(1,-1) \equiv S^{*}$, the class of starlike functions.

$M_{1}(1,-1) \equiv K$, the class of convex functions.

$M_{0}(A, B) \equiv S^{*}(A, B)$, the subclass of starlike functions studied by Goel et al. [2].

$M_{1}(A, B) \equiv K(A, B)$, the subclass of convex functions studied by Goel et al. [2].

For the complex sequence $a_{n}, a_{n+1}, a_{n+2}, \ldots$, the Hankel matrix, named after Herman Hankel (1839-1873), is the infinite matrix whose $(i, j)^{\text {th }}$ entry $a_{i j}$ is defined by

$$
a_{i j}=a_{n+i+j-2}(i, j, n \in N) \text {. }
$$

The $q^{\text {th }}$ Hankel matrix $(q \in \mathrm{N} \backslash\{1\})$ is by definition, the following $q$ $\mathrm{x} q$ square sub matrix:

$$
\left[\begin{array}{cccc}
a_{n} & a_{n+1} & \ldots & a_{n+q-1} \\
a_{n+1} & \ldots & \ldots & \ldots \\
\ldots & \ldots & \ldots & \ldots \\
a_{n+q-1} & \ldots & \ldots & a_{n+2 q-2}
\end{array}\right] .
$$

We observe that the Hankel matrix has constant positive slopping diagonals whose entries also satisfy:

$$
a_{i j}=a_{i-1, j+1}(i \in N \backslash\{1\} ; j \in N) \text {. }
$$

This also describes the Hankel matrix without reference to a particular sequence. The determinant of the $q^{\text {th }}$ Hankel matrix, usually denoted by

$$
H_{q}(n)=\left|\begin{array}{cccc}
a_{n} & a_{n+1} & \ldots & a_{n+q-1} \\
a_{n+1} & \ldots & \ldots & \ldots \\
\ldots & \ldots & \ldots & \ldots \\
a_{n+q-1} & \ldots & \ldots & a_{n+2 q-2}
\end{array}\right|,
$$

is called the $q^{\text {th }}$ Hankel determinant. In the particular cases

$$
q=2, n=1, a_{1}=1 \text { and } q=2, n=2,
$$

*Corresponding author: Gagandeep Singh, Department of Mathematics, M.S.K Girls College, Bharowal (Tarn-Taran), Punjab, India, Tel: 01655-221748; E-mail: kamboj.gagandeep@yahoo.in

Received May 24, 2014; Accepted June 23, 2014; Published June 27, 2014

Citation: Singh G, Singh G (2014) Second Hankel Determinant for a Subclass of Alpha Convex Functions. J Appl Computat Math 3: 167 doi:10.4172/21689679.1000167

Copyright: $\odot 2014$ Singh G, et al. This is an open-access article distributed under the terms of the Creative Commons Attribution License, which permits unrestricted use, distribution, and reproduction in any medium, provided the original author and source are credited. 
Citation: Singh G, Singh G (2014) Second Hankel Determinant for a Subclass of Alpha Convex Functions. J Appl Computat Math 3: 167 doi:10.4172/2168-9679.1000167

Page 2 of 3

the Hankel determinant simplifies respectively to

$$
H_{2}(1)=\left|a_{3}-a_{2}^{2}\right| \text { and } H_{2}(2)=\left|a_{2} a_{4}-a_{3}^{2}\right| \text {. }
$$

We refer to $H_{2}(2)$ as the second Hankel determinant. In 1976, Noonan et al., [3] stated the qth Hankel determinant of $f(z)$ for $q \geq 1$ and $n \geq 1$. After that Janteng et al. [4-6] Mehrok et al. [7], Singh [1,8] and many others have obtained sharp upper bounds of $H_{2}(2)$ for the classes of starlike functions, convex functions, alpha convex functions, starlike functions with respect to symmetric points, convex functions with respect to symmetric points etc.

For our discussion in this paper, we consider the second Hankel determinant and obtain an upper bound to the functional $H_{2}(2)$ for functions in the class $M_{\alpha}(A, B)$. Results due to Singh [1], Janteng et al. [5] and Singh and Singh [9] follows as special cases.

\section{Preliminary Results}

Let $P$ be the family of all functions $p$ analytic in $E$ for which $\operatorname{Re}(p(z))>0$ and

$$
p(z)=1+p_{1} z+p_{2} z^{2}+\ldots
$$

for $z \in E$.

Lemma 2.1.[9] If $p \in P$, then $\left|p_{k}\right| \leq 2(k=1,2,3, \ldots)$.

Lemma 2.2.[5,6] If $p \in P$, then

$$
\begin{aligned}
& 2 p_{2}=p_{1}^{2}+\left(4-p_{1}^{2}\right) x, \\
& 4 p_{3}=p_{1}^{3}+2 p_{1}\left(4-p_{1}^{2}\right) x-p_{1}\left(4-p_{1}^{2}\right) x^{2}+2\left(4-p_{1}^{2}\right)\left(1-|x|^{2}\right) z,
\end{aligned}
$$

for some $x$ and $z$ satisfying $|x| \leq 1,|z| \leq 1$ and $p_{1} \in[0,2]$.

\section{Main Result}

Theorem 3.1 If $f \in M_{\alpha}(A, B)$, then

$$
\left|a_{2} a_{4}-a_{3}^{2}\right| \leq \frac{(A-B)^{2}}{192(1+\alpha)(1+2 \alpha)^{2}(1+3 \alpha)}\left[\frac{(Q(\alpha))^{2}}{4 P(\alpha)}+48(1+\alpha)(1+3 \alpha)\right],
$$

where

$P(\alpha)=-\left\{\left(-2(1+2 \alpha)^{2}+3(1+\alpha)(1+3 \alpha)\right\} A^{2}+\left\{-12(1+2 \alpha)^{2}+12(1+\alpha)(1+3 \alpha)\right\} B^{2}+\left\{-12(1+\alpha)(1+3 \alpha)+10(1+2 \alpha)^{2}\right\} A B\right\}$

$+\left\{6(1+2 \alpha)^{2}-6(1+\alpha)(1+3 \alpha)\right\} A+\left\{-14(1+2 \alpha)^{2}+12(1+\alpha)(1+3 \alpha)\right\} B \mid+\left\{4(1+2 \alpha)^{2}-3(1+\alpha)(1+3 \alpha)\right\}$

and

$$
\begin{aligned}
& Q(\alpha)=4\left\{\left\{6(1+2 \alpha)^{2}-6(1+\alpha)(1+3 \alpha)\right\} A+\left\{-14(1+2 \alpha)^{2}+12(1+\alpha)(1+3 \alpha)\right\} B \mid\right. \\
& +4\left\{-3(1+\alpha)(1+3 \alpha)+4(1+2 \alpha)^{2}\right\}-12(1+\alpha)(1+3 \alpha) .
\end{aligned}
$$

Proof. If $f(z) \in M_{\alpha}(A, B)$, then there exists a Schwarz function $w(z) \in U$ such that

$$
\frac{z f^{\prime}(z)+\alpha z^{2} f^{\prime \prime}(z)}{(1-\alpha) f(z)+\alpha z f^{\prime}(z)}=\phi(w(z))
$$

where

$$
\begin{aligned}
& \phi(z)=\frac{1+A z}{1+B z}=1+(A-B) z-B(A-B) z^{2}+B^{2}(A-B) z^{3}+\ldots \\
& =1+B_{1} z+B_{2} z^{2}+B_{3} z^{3}+\ldots
\end{aligned}
$$

Define the function $p_{1}(z)$ by

$$
p_{1}(z)=\frac{1+w(z)}{1-w(z)}=1+c_{1} z+c_{2} z^{2}+c_{3} z^{3}+\ldots
$$

Since $w(z)$ is a Schwarz function, we see that $\operatorname{Re}\left(p_{1}(z)\right)>0$ and $p_{1}(0)=1$. Define the function $h(z)$ by

$$
h(z)=\frac{z f^{\prime}(z)+\alpha z^{2} f^{\prime \prime}(z)}{(1-\alpha) f(z)+\alpha z f^{\prime}(z)}=1+b_{1} z+b_{2} z^{2}+b_{3} z^{3}+\ldots
$$

In view of the equations (3.4), (3.6) and (3.7), we have

$$
\begin{aligned}
& h(z)=\phi\left(\frac{p_{1}(z)-1}{p_{1}(z)+1}\right)=\phi\left(\frac{c_{1} z+c_{2} z^{2}+c_{3} z^{3}+\ldots}{2+c_{1} z+c_{2} z^{2}+c_{3} z^{3}+\ldots}\right) \\
& =\phi\left(\frac{1}{2} c_{1} z+\frac{1}{2}\left(c_{2}-\frac{c_{1}^{2}}{2}\right) z^{2}+\frac{1}{2}\left(c_{3}-c_{1} c_{2}+\frac{c_{1}^{3}}{4}\right) z^{3}+\ldots\right) \\
& =1+\frac{B_{1} c_{1}}{2} z+\left[\frac{B_{1}}{2}\left(c_{2}-\frac{c_{1}^{2}}{2}\right)+\frac{B_{2} c_{1}^{2}}{4}\right] z^{2}+\left[\frac{B_{1}}{2}\left(c_{3}-c_{1} c_{2}+\frac{c_{1}^{3}}{4}\right)+\frac{B_{2} c_{1}}{2}\left(c_{2}-\frac{c_{1}^{2}}{2}\right)+\frac{B_{3} c_{1}^{3}}{8}\right] z^{3}+\ldots
\end{aligned}
$$

Thus,

$b_{1}=\frac{B_{1} c_{1}}{2} ; b_{2}=\frac{B_{1}}{2}\left(c_{2}-\frac{c_{1}^{2}}{2}\right)+\frac{B_{2} c_{1}^{2}}{4}$ and

$b_{3}=\frac{B_{1}}{2}\left(c_{3}-c_{1} c_{2}+\frac{c_{1}^{3}}{4}\right)+\frac{B_{2} c_{1}}{2}\left(c_{2}-\frac{c_{1}^{2}}{2}\right)+\frac{B_{3} c_{1}^{3}}{8}$.

Using (3.5) and (3.7) in (3.8), we obtain

$$
\left.\begin{array}{l}
a_{2}=\frac{(A-B) c_{1}}{2(1+\alpha)}, \\
a_{3}=\frac{(A-B)}{8(1+2 \alpha)}\left[2 c_{2}+(A-2 B-1) c_{1}^{2}\right], \\
a_{4}=\frac{(A-B)}{48(1+3 \alpha)}\left[8 c_{3}+(6 A-14 B-8) c_{1} c_{2}+\left(A^{2}+6 B^{2}-5 A B-3 A+7 B+2\right) c_{1}^{3}\right]
\end{array}\right\} .
$$

(3.9) yields,

$$
a_{2} a_{4}-a_{3}^{2}=\frac{(A-B)^{2}}{C(\alpha)}\left\{L c_{1}\left(4 c_{3}\right)+M c_{1}^{2}\left(2 c_{2}\right)+N c_{1}^{4}-R\left(4 c_{2}^{2}\right)\right\}
$$

where $\quad C(\alpha)=192(1+\alpha)(1+2 \alpha)^{2}(1+3 \alpha)$,

$L=4(1+2 \alpha)^{2}$,

$M=\left\{6(1+2 \alpha)^{2}-6(1+\alpha)(1+3 \alpha)\right\} A+\left[12(1+\alpha)(1+3 \alpha)-14(1+2 \alpha)^{2}\right] B+\left[6(1+\alpha)(1+3 \alpha)-8(1+2 \alpha)^{2}\right]$

$N=\left\{2(1+2 \alpha)^{2}-3(1+\alpha)(1+3 \alpha)\right\} A^{2}+\left[12(1+2 \alpha)^{2}-12(1+\alpha)(1+3 \alpha)\right] B^{2}+\left[12(1+\alpha)(1+3 \alpha)-10(1+2 \alpha)^{2}\right] A B$ $+\left\{-6(1+2 \alpha)^{2}+6(1+\alpha)(1+3 \alpha)\right\} A+\left[14(1+2 \alpha)^{2}-12(1+\alpha)(1+3 \alpha)\right] B+\left[4(1+2 \alpha)^{2}-3(1+\alpha)(1+3 \alpha)\right]$

and

$$
R=3(1+3 \alpha)(1+\alpha) .
$$

Using Lemma 2.1 and Lemma 2.2 in (3.10), we obtain

$$
\left|a_{2} a_{4}-a_{3}^{2}\right|=\frac{(A-B)^{2}}{C(\alpha)} \mid \begin{aligned}
& {\left[\begin{array}{l}
\left\{2(1+2 \alpha)^{2}-3(1+\alpha)(1+3 \alpha)\right\} A^{2}+\left\{12(1+2 \alpha)^{2}-12(1+\alpha)(1+3 \alpha)\right\} B^{2} \\
+\left\{12(1+\alpha)(1+3 \alpha)-10(1+2 \alpha)^{2}\right\}
\end{array}\right] c_{1}^{4}} \\
& +\left[\left\{\begin{array}{l}
\left.\left[6(1+2 \alpha)^{2}-6(1+\alpha)(1+3 \alpha)\right\} A+\left\{-14(1+2 \alpha)^{2}+12(1+\alpha)(1+3 \alpha)\right\} B\right] c_{1}^{2} x\left(4-c_{1}^{2}\right) \\
-\left[12(1+\alpha)(1+3 \alpha)+\left\{4(1+2 \alpha)^{2}-3(1+\alpha)(1+3 \alpha)\right\} c_{1}^{2}\right] x^{2}\left(4-c_{1}^{2}\right) \\
+8(1+2 \alpha)^{2} c_{1}\left(4-c_{1}^{2}\right)\left(1-|x|^{2}\right) z
\end{array}\right.\right.
\end{aligned}
$$

Assume that $c_{1}=c$ and $c \in[0,2]$, using triangular inequality and $|z| \leq 1$, we have 
Citation: Singh G, Singh G (2014) Second Hankel Determinant for a Subclass of Alpha Convex Functions. J Appl Computat Math 3: 167 doi:10.4172/2168-9679.1000167

$$
\left|a_{2} a_{4}-a_{3}^{2}\right| \leq \frac{(A-B)^{2}}{C(\alpha)}\left[\begin{array}{l}
\mid \begin{array}{l}
\left\{-2(1+2 \alpha)^{2}+3(1+\alpha)(1+3 \alpha)\right\} A^{2}+\left\{-12(1+2 \alpha)^{2}+12(1+\alpha)(1+3 \alpha)\right\} B^{2} \mid \\
+\left\{-12(1+\alpha)(1+3 \alpha)+10(1+2 \alpha)^{2}\right\} A B \\
+8(1+2 \alpha)^{2} c\left(4-c^{2}\right)
\end{array} \\
+\left|\left\{6(1+2 \alpha)^{2}-6(1+\alpha)(1+3 \alpha)\right\} A+\left\{-14(1+2 \alpha)^{2}+12(1+\alpha)(1+3 \alpha)\right\} B\right| c^{2}\left(4-c^{2}\right) \delta \\
+\left[\left[12(1+\alpha)(1+3 \alpha)+\left\{4(1+2 \alpha)^{2}-3(1+\alpha)(1+3 \alpha)\right\} c^{2}\right]-8(1+2 \alpha)^{2} c\left(4-c^{2}\right)\right] \delta^{2}
\end{array}\right]
$$

$=\frac{(A-B)^{2}}{C(\alpha)} F(\delta)$, where $\delta=|x| \leq 1$ and

$F(\delta)=\left\{\begin{array}{l}\left\{-2(1+2 \alpha)^{2}+3(1+\alpha)(1+3 \alpha)\right\} A^{2}+\left\{-12(1+2 \alpha)^{2}+12(1+\alpha)(1+3 \alpha)\right\} B^{2} \\ +\left\{-12(1+\alpha)(1+3 \alpha)+10(1+2 \alpha)^{2}\right\} A B\end{array} \mid c^{4}\right.$

$+8(1+2 \alpha)^{2} c\left(4-c^{2}\right)+\left|\left\{6(1+2 \alpha)^{2}-6(1+\alpha)(1+3 \alpha)\right\} A+\left\{-14(1+2 \alpha)^{2}+12(1+\alpha)(1+3 \alpha)\right\} B\right| c^{2}\left(4-c^{2}\right) \delta$

$+\left[\left[12(1+\alpha)(1+3 \alpha)+\left\{4(1+2 \alpha)^{2}-3(1+\alpha)(1+3 \alpha)\right\} c^{2}\right]-8(1+2 \alpha)^{2} c\left(4-c^{2}\right)\right] \delta^{2}$

is an increasing function. Therefore $\operatorname{Max} . F(\delta)=F(1)$.

Consequently

$\left|a_{2} a_{4}-a_{3}^{2}\right| \leq \frac{(A-B)^{2}}{C(\alpha)} G(c)$,

where

$G(c)=F(1)$.

So $G(c)=-P(\alpha) c^{4}+Q(\alpha) c^{2}+48(1+\alpha)(1+3 \alpha)$,

where $P(\alpha)$ and $Q(\alpha)$ are defined in (3.2) and (3.3) respectively.

Now $G^{\prime}(c)=-4 P(\alpha) c^{3}+2 Q(\alpha) c$

and $G^{\prime \prime}(c)=-12 P(\alpha) c^{2}+2 Q(\alpha)$.

$G^{\prime}(c)=0$ gives

$c\left\{-4 P(\alpha) c^{2}+2 Q(\alpha)\right\}=0$.

$G^{\prime \prime}(c)$ is negative at $c=\sqrt{\frac{Q(\alpha)}{2 P(\alpha)}}=c^{\prime}$.

So $\operatorname{Max} . G(c)=G\left(c^{\prime}\right)$.

Hence from (3.11), we obtain (3.1).

The result is sharp for $c_{1}=c^{\prime}, c_{2}=c_{1}^{2}-2$ and $c_{3}=c_{1}\left(c_{1}^{2}-3\right)$.

For $A=1$ and $B=-1$, Theorem 3.1 gives the following result due to Singh [1].

Corollary 3.1.1 If $f(z) \in M(\alpha)$, then

$$
\left|a_{2} a_{4}-a_{3}^{2}\right| \leq \frac{1}{(1+\alpha)(1+3 \alpha)} \text {. }
$$
al. [9].

For $\alpha=0$, Theorem 3.1 gives the following result due to Singh et

Corollary 3.1.2 If $f(z) \in S^{*}(A, B)$, then

$$
\left|a_{2} a_{4}-a_{3}^{2}\right| \leq \frac{(A-B)^{2}}{4} \text {. }
$$

For $\alpha=1$, Theorem 3.1 gives the following result due to Singh et al. [9].

Corollary 3.1.3 If $f \in K(A, B)$, then

$$
\left|a_{2} a_{4}-a_{3}^{2}\right| \leq \frac{(A-B)^{2}}{576}\left[\frac{16\left|-A^{2}+2 B^{2}+A B\right|-|A-5 B|^{2}-12|A-5 B|-36}{\left|-A^{2}+2 B^{2}+A B\right|-|A-5 B|-2}\right] .
$$

Putting $\alpha=0, A=1$ and $B=-1$ in Theorem 3.1, we obtain the following result due to Janteng et al. [5].

Corollary 3.1.4 If $f(z) \in S^{*}$, then

$\left|a_{2} a_{4}-a_{3}^{2}\right| \leq 1$.

Putting $\alpha=1, A=1$ and $B=-1$ in Theorem 3.1, we obtain the following result due to Janteng et al. [5].

Corollary 3.1.5 If $f(z) \in K$, then

$$
\left|a_{2} a_{4}-a_{3}^{2}\right| \leq \frac{1}{8}
$$

\section{Conclusion}

The results obtained in this paper are very useful as many results already proved by various authors can be easily obtained from these results by giving particular values to $A$ and $B$ as shown in above corollaries. Also these results can provide some new ideas to future researchers for further study of this topic.

\section{References}

1. Singh G (2012) Hankel determinant for a new subclass of analytic functions. Scientia Magna 8: 61-65.

2. Goel RM, Mehrok BS (1981) On the coefficients of a subclass of starlike functions. Ind J Pure and Appl Math 12: 634-647.

3. Noonan JW, Thomas DK (1976) On the second Hankel determinant of a really mean p-valent functions. Trans Amer Math Soc 223: 337-346.

4. Janteng A, Halim AS, Maslina D (2006) Coefficient inequality for a function whose derivative has a positive real part J Ineq Pure Appl Math 7: 50.

5. Janteng A, Halim SA, Darus M (2007) Hankel determinant for starlike and convex functions. Int J Math Anal 1: 619-625.

6. Janteng A, Halim SA, Darus M (2006) Hankel determinant for functions starlike and convex with respect to symmetric points. J Quality Measurement and Anal 2: $37-43$.

7. Mehrok BS, Singh G (2012) Estimate of second Hankel determinant for certain classes of analytic functions. Scientia Magna 8: 85-94.

8. Singh G (2013) Hankel determinant for new subclasses of analytic functions with respect to symmetric points. Int $\mathrm{J}$ of Modern Math Sci 5: 67-76.

9. Singh G, Singh G, Second Hankel determinant for subclasses of starlike and convex functions. 